\title{
THE EFFECTS OF SCATTERING ON SOLAR OSCILLATIONS
}

\author{
Peter Goldreich and Norman Murray \\ California Institute of Technology, Pasadena, CA 91125 \\ Received 1993 March 31; accepted 1993 September 16
}

\begin{abstract}
Acoustic modes are scattered by turbulent velocity fluctuations in the solar convection zone. The strongest scattering occurs near the top of the acoustic cavity where the mode changes character from propagating to evanescent. This layer is located at depth $z_{1} \sim g / \omega^{2}$ below the photosphere. The scattering optical depth $\tau_{s}$ is of order $\mathscr{M}_{1}^{2}$, where $\mathscr{M}_{1}$ is the Mach number of the energy-bearing eddies at $z_{1}$. The corresponding contribution to the line width is $\gamma^{s} \sim \omega \mathscr{M}_{1}^{2} / \pi(n+1)$, where $n$ is the mode's radial order. At the top of the acoustic cavity the correlation time of energy-bearing eddies is much longer than $\omega^{-1}$. Also, the pressure scale height $H$ and the eddy correlation length $\Lambda$ are comparable to $\omega / c$, where $c$ is the sound speed. Thus scattering couples modes of similar $\omega$ and all $\ell$ and has little effect on the sum of their energies. Observations show that mode energies decline with decreasing $n$ (increasing $\ell$ ) at fixed $\omega$. Consequently, scattering damps $p$-modes and excites $f$-modes.
\end{abstract}

Subject headings: convection - Sun: interior - Sun: oscillations

\section{INTRODUCTION}

We examine the rate at which scattering by turbulent convection transfers energy among solar $p$-modes and $f$-modes. An order-of-magnitude calculation of $\gamma^{s}$, the radian line width due to scattering, is presented in $\S 2$. Details of a more complete calculation are relegated to the Appendix. Next, in $\S 3$, we compare our estimate of $\gamma^{s}$ with an estimate of the absorptive line width, $\gamma^{a}$, derived by Goldreich \& Kumar (1991). A comparison of the theoretically and observationally determined line widths is the subject of $\S 4$.

\section{ESTIMATE OF SCATTERING LINE WIDTH}

\subsection{Scattering in an Infinite Medium}

We analyze a simple example of the scattering of a linear acoustic wave by a turbulent velocity field. Except for a single layer of eddies centered on the plane $z=0$, the background medium is taken to be static and homogeneous. The incident wave is plane-parallel, harmonic, and propagates in the positive $z$-direction. The turbulent velocity, $v_{\Lambda}$, is assumed to be much smaller than the adiabatic sound speed $c$. We assume the eddies have a correlation length $\Lambda$ that is much larger than the wavelength, $\lambda=2 \pi / k$, and a correlation time $\tau_{\Lambda}$ that is much longer than the wave period, $P=2 \pi / \omega$. Geometrical optics then applies. We assume further that the scattering is weak in the sense that the extra phase shift induced by propagation through the eddies is small. Under these conditions the layer of eddies acts like a thin, phase-changing screen.

The incident and transmitted waves may be written as

for $z \lesssim-\Lambda$, and

$$
q_{i}=A \exp i(\omega t-k z)
$$

$$
q_{t} \approx A \exp i(\omega t-k z+\Delta \phi)
$$

for $\Lambda \lesssim z \ll k \Lambda^{2}$. Here $q$ denotes the perturbation in any dependent variable, and $\Delta \phi(x, y)$ is the phase shift induced by the eddies. In the geometrical optics limit, the wave front propagates relative to the fluid at the speed of sound. Thus

$$
\Delta \phi \approx k \int d z \frac{v_{z}}{c},
$$

with $v_{z}=O\left(v_{\Lambda}\right)$ the $z$ component of the velocity field. Clearly, the magnitude of $\Delta \phi$ is of order $k \Lambda v_{\Lambda} / c$, and its spatial variation takes place on scale $\Lambda$. The scattered wave, $q_{s} \approx q_{t}-q_{i}$, may then be written as

$$
q_{s} \approx \frac{i A}{2 \pi} \int d^{2} k_{h} \Delta \phi_{k_{h}} \exp i\left(\omega t-k_{h} \cdot x-k_{z} z\right),
$$

where

$$
\Delta \phi_{k_{h}} \equiv \frac{1}{2 \pi} \int d^{2} x \Delta \phi \exp i\left(\boldsymbol{k}_{h} \cdot x\right),
$$

and $k_{z}^{2}+k_{h}^{2}=k^{2}$. The two-dimensional, spatial power spectrum of $\Delta \phi(x, y ; t)$ is compact, with much of the power confined within $k_{h} \equiv\left(k_{x}^{2}+k_{y}^{2}\right)^{1 / 2} \lesssim 1 / \Lambda$.

Because we are concerned with weak scattering, the scattering optical depth may be defined as the ratio of the power in the scattered wave to that in the incident wave. Hence, the scattering optical depth ${ }^{1}$ is

$$
\tau_{s} \sim(k \Lambda)^{2} \mathscr{M}^{2},
$$

where $\mathscr{M}=v_{\Lambda} / c$ is the Mach number that characterizes the turbulent velocity field. The scattering angle is given by sin $\theta_{s}=\left|k_{h}\right| / k$, so the scattered waves are beamed in a cone with opening angle

$$
\Delta \theta_{s} \sim \frac{1}{k \Lambda} \ll 1 .
$$

The scattered waves suffer frequency shifts of order

$$
\Delta \omega=\frac{\partial \Delta \phi_{\boldsymbol{k}_{h}}}{\partial t} \sim \frac{1}{\tau_{\Lambda}} .
$$

\subsection{Scattering in a Box}

We turn next to a subtle issue regarding the distinction between the scattering of acoustic waves and acoustic modes. Imagine enclosing the medium described above in a cubical box with sides of length $L \geq \Lambda$. The acoustic modes satisfy the

\footnotetext{
${ }^{1}$ Note the distinction between the optical depth $\tau_{s}$ and the eddy correlation time $\tau_{\Lambda}$
} 
dispersion relation

$$
\omega=\left(\ell^{2}+m^{2}+n^{2}\right)^{1 / 2} \frac{\pi c}{L},
$$

where $(\ell, m, n)$ are a triad of integers. The number density of modes is

$$
\frac{d N}{d \omega} \approx \frac{\omega^{2} L^{3}}{2 \pi^{2} c^{3}}
$$

For simplicity, we assume that the mode line widths are due entirely to scattering, and that the sizes of the eddies match the wavelengths of the modes under consideration in such a way that $k \Lambda \sim 1$. $^{2}$

A mode having $\ell=m=0$ and $n>0$ (referred to as mode $\alpha$ ) can be decomposed into a properly phased pair of waves traveling in opposite directions parallel to the $z$-axis. Scattering couples mode $\alpha$ to all modes $\beta$ that satisfy

$$
\left|\omega_{\beta}-\omega_{\alpha}\right| \lesssim \frac{1}{\tau_{\Lambda}}
$$

The number of such modes $\beta$ is

$$
\Delta N \approx \frac{d N}{d \omega} \frac{1}{\tau_{\Lambda}} \sim\left(\frac{L}{\Lambda}\right)^{3} \frac{\mathscr{M}}{2 \pi^{2}} .
$$

Let us assume that $\Delta N \gg 1$, so that there are many accessible final-state modes $\beta$. Then, since a fraction $f \approx 2 / n$ of mode $\alpha$ 's energy passes through the eddy layer each period, and the layer has $\tau_{s} \sim \mathscr{M}^{2}$, the scattering line width is

$$
\gamma^{s} \sim \frac{\omega}{\pi n} \mathscr{M}^{2}
$$

Scattering tends to equalize the actions of the modes it couples. Action is proportional to energy divided by frequency; it is the classical equivalent of the number of quanta. In the limit that $\omega_{\alpha} \tau_{\Lambda} \ll 1$, the scattering is elastic and action conservation reduces to energy conservation.

Suppose there are no final states available to receive energy scattered out of mode $\alpha$-that is, $\Delta N \ll 1$. Mode $\alpha$ still exchanges energy with each mode $\beta$, but how now it is in a quasi-periodic fashion at frequency $\left|\omega_{\alpha}-\omega_{\beta}\right|$. These exchanges cause the energy of mode $\alpha$ to fluctuate in such a way that

$$
\frac{\Delta E_{\alpha}}{E_{\alpha}} \sim n^{2} \mathscr{M}^{2} .
$$

The fluctuations are dominated by those modes $\beta$ whose frequencies lie closest to that of mode $\alpha$. Since no further use is made of equation (14), we have its derivation to the reader. Suffice it to say that one can be constructed from equation (A20).

\subsection{Application to Solar $p$-Modes}

The relations for $\Delta \theta_{s}$ and $\gamma^{s}$, interpreted as WKB approximations, can be applied to the solar $p$-modes. We assume that there are always final states available to receive the scattered energy. In $\S 4$ we show that this is true for all $\omega$ at which $\gamma$ has been determined to date. We take $\Lambda \sim H$, where $H$ is the local pressure scale height:

$$
H \sim c^{2} / g,
$$

\footnotetext{
${ }^{2}$ The latter condition pushes geometrical optics somewhat beyond its limit of validity. Here and in what follows, this procedure is justified by the results obtained in the Appendix.
}

with $g$ the magnitude of the local gravitational acceleration. The product $k H$ increases with depth in the convection zone:

$$
k H=\frac{\omega H}{c} \sim \frac{\omega c}{g} .
$$

The dependence of $\tau_{s}$ on depth follows from equations (6) and $(16):^{3}$

$$
\tau_{s} \sim\left(\frac{\omega v_{H}}{g}\right)^{2}
$$

We estimate the convective velocity, $v_{H}$, from the mixinglength relation

$$
F \sim \rho v_{\mathrm{H}}^{3},
$$

where $F$ is the convective flux and $\rho$ is the mass density. All this shows that $\tau_{s} \propto \rho^{-2 / 3}$, at least where $k H \gg 1$. In other words, the strength of the scattering increases with height in regions where geometrical optics applies. Pushing equations (7) and (6) to the limits of their validity, i.e., $k H \rightarrow 1$, we deduce that

$$
\Delta \theta_{s} \sim 1
$$

and

$$
\tau_{s} \sim \mathscr{M}_{1}^{2} .
$$

Here $\mathscr{M}_{1}$ is the Mach number evaluated where $k H \sim 1$; this contribution serves to define the top of the acoustic cavity. ${ }^{4}$ Note that

$$
H_{1} \sim g / \omega^{2} .
$$

As in the simpler example of the homogeneous box, the scattering line width is given by

$$
\gamma^{s} \sim \frac{\omega}{\pi} \frac{\mathscr{M}_{1}^{2}}{(n+1)} .
$$

The 1 added to $n$ accounts, in crude fashion, for the energy stored above the top of the acoustic cavity.

In accepting equation (22), we are making the implicit assumption that higher layers, in which $k H \ll 1$, make negligible contributions to $\tau_{s}$. The validity of this assumption is demonstrated in the Appendix. Here we present a more heuristic discussion identifying the main points. Sticking for the moment to a homogeneous medium, we note that, for $k H \ll 1$,

$$
\tau_{s} \sim(k H)^{4} \mathscr{M}^{2},
$$

and that comparable amounts of power are scattered in the forward and backward directions (Goldreich \& Kumar 1988). There is an extra factor of $(k H)^{2}$ in the expression for $\tau_{s}$ as compared with that which applies in the geometrical optics limit. It appears because only that portion of the twodimensional power spectrum of $\int d z v_{z}$ corresponding to $k_{h} \lesssim$ $\omega / c$ contributes to the scattered flux. For uncorrelated eddies, the power in this part of the spectrum is a fraction $(k H)^{2} \ll 1$ of the whole. The other new features are associated with the effects of stratification. Only the evanescent tails of acoustic modes penetrate into regions where $k_{z} H \ll 1$. This results in a sharp reduction in $\tau_{s}$ below the value given by equation (23).

\section{SCATTERING VERSUS ABSORPTION}

Scattering is not the only source of $p$-mode line widths. Nonadiabatic effects associated with radiative and convective energy transport, and the mechanical absorption of sound

\footnotetext{
${ }^{3}$ As defined here, $\tau_{s}$ is the optical depth contributed by a single layer of scale-height-size eddies.

${ }^{4}$ We use a subscript 1 to denote quantities evaluated at this level.
} 
waves by turbulence, also act as sources for the line widths (Ando \& Osaki 1975; Goldreich \& Keeley 1977; Antia, Chitre, \& Narasimha 1986; Christensen-Dalsgaard, Gough, \& Libbrecht 1989; Balmforth 1992a, b). How do the contributions of these absorptive mechanisms compare with that due to scattering?

The comparison is simplified because thermal and mechanical absorption make comparable contributions to the absorptive line width, $\gamma^{a}$. This remarkable occurrence, implicit in the numerical results of Goldreich \& Keeley (1977) and Balmforth (1992a), is elucidated by Goldreich \& Kumar (1991). The latter authors derive a simple expression for the absorptive contribution to the line width, $\gamma^{a}$, that agrees well with the above-cited numerical results for $v \lesssim 3 \mathrm{mHz}$. It is

$$
\gamma^{a} \sim \frac{L_{\odot}}{M_{\alpha} c_{t}^{2}}\left(\frac{\omega}{\omega_{\mathrm{ac}}}\right)^{2},
$$

where $L_{\odot}$ is the solar luminosity; $c_{t}$ is the sound speed at the top of the convection zone; $\omega_{\mathrm{ac}} \sim g / c_{t}$ is the acoustic cutoff frequency, the highest frequency at which sound waves are trapped in the Sun; and $M_{\alpha}$ is the mass of mode $\alpha$.

The mode mass is the coefficient of proportionally between the mode's energy and the average value of the square of its surface velocity.

$$
M_{\alpha} \equiv \frac{4 \pi}{\omega_{\alpha}^{2} \int d \Omega\left|\xi_{\alpha}\left(R_{\odot}, \theta, \phi\right)\right|^{2}},
$$

for a mode whose displacement eigenfunction, $\xi_{\alpha}$, is normalized such that

$$
\omega^{2} \int d^{3} x \rho \xi_{\alpha} \cdot \xi_{\alpha}^{*}=1
$$

There is a convenient analytic approximation for the mode mass. It involves three ingredients. The first is the WKB flux relation for the envelope of the angle-averaged mean square displacement, $\left\langle\left|\xi_{\alpha}\right|^{2}\right\rangle$, namely,

$$
r^{2} \rho c\left\langle\left|\xi_{\alpha}\right|^{2}\right\rangle \approx \text { constant }
$$

which holds between the upper and lower boundaries of the acoustic cavity at $r_{1}$ and $r_{2}$. The second is the period relation

$$
\int_{r_{2}}^{r_{1}} \frac{d r}{c} \approx \frac{\pi(n+1)}{\omega} .
$$

The third is the ratio

$$
f \equiv \frac{\left\langle\left|\xi_{\alpha}\right|^{2}\right\rangle_{1}}{\left\langle\left|\xi_{\alpha}\right|^{2}\right\rangle_{R_{\odot}}} \leq 1 .
$$

Putting these ingredients together, we obtain

$$
M_{\alpha} \approx \frac{4 \pi^{2} f R_{\odot}^{2}(n+1) \rho_{1} c_{1}}{\omega} .
$$

Next we recast $\gamma^{a}$ given by equation (24) using equations (15), (18), (21), and (30), so that it reads

$$
\gamma^{a} \sim \frac{\omega}{\pi f} \frac{\mathscr{M}^{3}}{(n+1)} .
$$

The ratio of $\gamma^{a}$ to $\gamma^{s}$ takes the form

$$
\frac{\gamma^{a}}{\gamma^{s}} \sim \frac{\mathscr{M}_{1}}{f}
$$

This ratio assumes a particularly revealing form when evaluated for a plane-parallel model atmosphere whose parameters are chosen so that it resembles the solar envelope. The atmosphere is subject to a constant gravitational acceleration $g$, and consists of two layers, the lower an isentropic polytrope of index $m$ and the upper isothermal. The coordinate $z$ measures depth from the level that would form the top of the polytropic layer were the isothermal layer not present. The upper and lower layers are joined at $z_{t}$. Then $c_{1} / c_{t} \sim\left(g / \omega^{2} z_{t}\right)^{1 / 2}$ and $\rho / \rho_{t} \sim\left(g / \omega^{2} z_{t}\right)^{m}$. With the aid of equation (18), we arive at

$$
\frac{\gamma^{a}}{\gamma^{s}} \sim \frac{\mathscr{M}_{t}}{f}\left(\frac{\omega}{\omega_{\mathrm{ac}}}\right)^{1+2 m / 3} .
$$

Our crude derivation suggests that scattering dominates absorption for $\omega \ll \omega_{\text {ac }}$.

\section{COMPARISON WITH OBSERVED LINE WIDTHS}

\subsection{Scattering Rates}

Observations by Libbrecht (1988) established $\gamma \propto v^{4.2}$, except for a plateau near $v \approx 3 \mathrm{mHz}$. By contrast, theoretical absorption line widths exhibit a much steeper frequency dependence, $\gamma^{\alpha} \propto v^{8}$, for $v \lesssim 3 \mathrm{mHz}$, and flatten out at higher $v$ (Goldreich \& Keeley 1977; Balmforth 1992a). These facts, and the discussion in $\S 3$, suggest that scattering may contribute most of the line width except near $3 \mathrm{mHz}$, where absorption is likely to be of comparable importance.

Here we apply the machinery developed in the Appendix to make a quantitative assessment of the scattering line width. We start with some definitions. The exchange of phonons by scattering between modes $\alpha$ and $\beta$ contributes an amount $\gamma_{\alpha \beta}^{s}$ to each mode's line width. Mode $\alpha$ 's total scattering line width is given by

$$
\gamma_{\alpha}^{s}=\sum_{\beta} \gamma_{\alpha \beta}^{s},
$$

where the sum extends over all modes $\beta$. We accomplish the sum in two steps, first over all modes with radial order $n_{\beta}$ and then over all $n_{\beta}$. Thus

$$
\gamma_{\alpha}^{s}=\sum_{n \beta} \gamma_{\alpha \beta}^{s} \Delta N_{\beta}
$$

where (cf. eq. [A24])

$$
\begin{aligned}
\gamma_{\alpha \beta}^{s} \Delta N_{\beta} \approx & 16 \pi^{2} R_{\odot}^{4} \omega^{4} k_{\beta} \frac{d k_{\beta}}{d \omega} \\
& \times \int_{r_{b}}^{R_{\odot}} d \bar{r} \rho^{2} v_{\Lambda}^{2} \mathscr{S}^{2} \Lambda^{2} \exp \left[-\frac{\left(k_{\beta} \mathscr{S} \Lambda\right)^{2}}{4 \pi}\right] \\
& \times \int_{\Delta r_{\ell}}^{\Delta r_{u}} d \Delta r \frac{\partial \tilde{\xi}_{\alpha}^{r^{\prime}}}{\partial r^{\prime}} \frac{\partial \tilde{\xi}_{\alpha}^{\prime \prime}}{\partial r^{\prime \prime}} \tilde{\xi}_{\beta}^{r^{\prime}} \tilde{\xi}_{\beta}^{r^{\prime \prime}} \exp \left[-\pi\left(\frac{\Delta r}{\Lambda}\right)^{2}\right] .
\end{aligned}
$$

Here $r^{\prime}=\bar{r}-\Delta r / 2$ and $r^{\prime \prime}=\bar{r}+\Delta r / 2$. The upper and lower limits on the integral over $\Delta r$ are $\pm 2\left(\bar{r}-r_{b}\right)$ if $r_{b} \leq \bar{r} \leq\left(r_{b}\right.$ $\left.+R_{\odot}\right) / 2$, and $\pm 2\left(R_{\odot}-\bar{r}\right)$ if $\left(r_{b}+R_{\odot}\right) / 2 \leq \bar{r} \leq R_{\odot}$, where $r_{b}$ denotes the base of the convection zone. The upper sign refers to the upper limit, the lower sign to the lower limit. The variables $\Lambda$ and $v_{\Lambda}$ are the mixing length and mixing-length velocity. The aspect ratio $\mathscr{S}$ describes the ratio of the width to the height of the energy-bearing eddies. The horizontal wavevector $\boldsymbol{k}$ is related to the angular degree $\ell$ by $k \equiv \ell / R_{\odot}$. Equation (36) applies for $\ell_{\alpha} \ll \ell_{\beta}$.

We evaluate $\gamma_{\alpha \beta}^{s}$ numerically using $\rho, \Lambda, v_{\Lambda}$, and $\xi$ obtained from the solar model of Christensen-Dalsgaard (1982). Our calculations include the $f$-mode ridge and the 30 lower $p$-mode ridges in the $k$ - $\omega$ diagram. Figure 1 displays $\gamma^{s}(v)$ for modes with $\ell=0$ as obtained by setting $\mathscr{S}=4$ (solid line). The points 


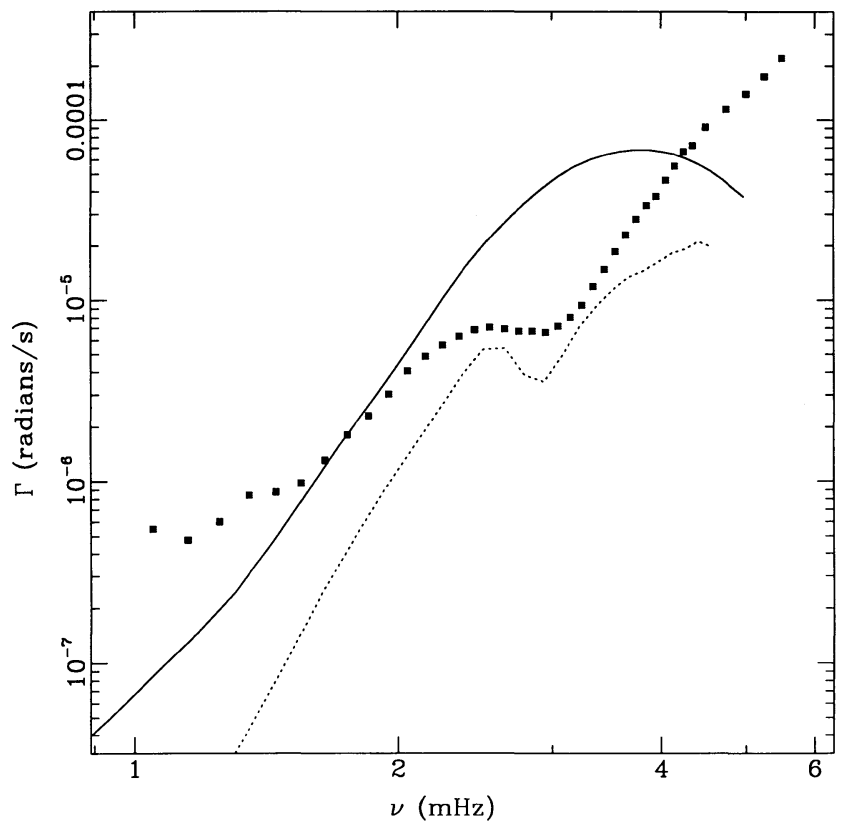

FIG. 1.-Low- $\ell$ solar $p$-mode decay rates as a function of frequency. The points are from the observations of Libbrecht \& Woodard (1991). The lowfrequency results are consistent with a power law, $\Gamma \sim v^{4}$; the finite observation time (about 5 months) can account for the apparent deviation from a power law below $v \approx 1.4 \mathrm{mHz}$. The solid line is the result of integrating eq. (36) with $\mathscr{S}=4$. The dotted line shows the line width calculated by Balmforth (1992a).

are the line widths measured by Libbrecht \& Woodard (1991). The calculated $\gamma^{s}(v)$ does not provide a close fit to the observationally determined $\gamma(v)$. It fails to reproduce the plateau near $v \approx 3 \mathrm{mHz}$. Also, its slope is too steep at low frequencies. This latter deficiency, while serious, is considerably less pronounced than that exhibited by calculated $\gamma^{a}(v)$. For example, we show in Figure $\gamma^{a}(v)$ as given in Table 1 of Balmforth (1992a).

In Figure 2 we plot the integrand of the expression for $\gamma_{\alpha \beta}^{s} \Delta N_{\beta}$ as a function of the pressure $p$ for $v_{\alpha}=3 \mathrm{mHz}, \ell_{\alpha}=0$, and several different choices for $n_{\beta}$. Note that mode $\alpha$ is most strongly coupled to the $f$-mode ridge. By summing such integrands over all $n_{\beta}$, we obtain the integrands for $\gamma_{\alpha}^{s}$ that are displayed in Figure 3. The figure confirms that most of the scattering occurs near the top of mode $\alpha$ 's acoustic cavity. This layer is located near the bottom of the convection zone for $v \approx 0.5 \mathrm{mHz}$ and near the top for $v \approx 5 \mathrm{mHz}$. The solid line gives the summed integrand, while the dashed line shows the contribution from scattering into the $f$-mode only.

The figure for $v_{\alpha}=3 \mathrm{mHz}$ is of particular interest, since it is near this frequency that the measured line widths deviate from a power law. Near $3 \mathrm{mHz}$, the integrand for $\gamma_{\alpha}^{s}$ peaks in the hydrogen ionization zone where $10^{6} \lesssim p \lesssim 10^{7}$ dynes $\mathrm{cm}^{-2}$. As illustrated in Figure 4, this corresponds to the depth at which the effective polytropic index $m$, defined by $\Gamma=\rho c^{2} /$ $p=1+1 / m$, is largest. Perhaps the ionization affects the convection in a manner not captured by the mixing-length Ansatz - for example, by reducing either the magnitude or the correlation length of the turbulent velocity.

The inadequacies of mixing-length theory are well documented. In the form we apply, the mixing length is a local parameter, but the convective dynamics is certainly not local. Also, the enthalpy flux is taken to be the entire convective flux; this ignores the comparable contribution made by the flux of

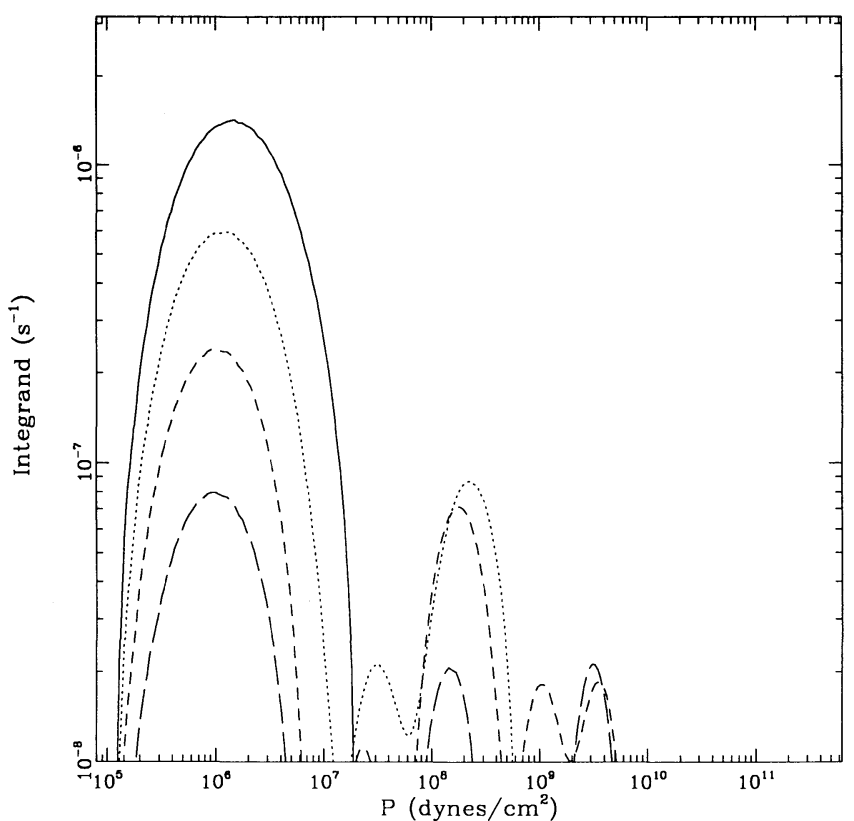

Fig. 2.- Integrand in eq. (36) for the scattering line width at $v=3.05 \mathrm{mHz}$ for different final-state modes $n_{\beta} ; n_{\beta}=0$ (solid line), $n_{\beta}=1$ (dotted line), $n_{\beta}=2$ (short-dashed line), and $n_{\beta}=3$ (long-dashed line).

kinetic energy. The relative importance of the kinetic energy flux drops in ionization zones where the specific heat is large (Rast et al. 1993). This might account for the dip in the line widths that our calculations fail to reproduce. Above all, numerical simulations (Cattaneo et al. 1991; Stein \& Nordlund 1989) of convection reveal qualitative features that are outside the purview of the mixing-length model. In particular, they exhibit striking asymmetries between broad, slowly rising warm plumes and narrow columns of cold, fast downflows.

\subsection{Availability of Final States}

Scattering couples modes whose frequencies differ by up to the inverse of the correlation time of the turbulent velocity field, $\Delta \omega \lesssim 1 / \tau_{H} \sim v_{H} / H$. The $f$-modes are the principal finalstate scattering partners of $p$-modes. Scattering between a $p$-mode and an $f$-mode is concentrated near the top of the $p$-mode's acoustic cavity where $\omega H / c \sim 1 .^{5}$ Thus the frequency shift associated with scattering is

$$
\Delta v_{s} \approx \mathscr{M}_{1} v \text {. }
$$

The availability of final states requires that the frequency spacing between consecutive $f$-modes be smaller than $\Delta v_{s}$. Rotation complicates this issue by lifting the $m$-degeneracy among modes of identical $n$ and $\ell$.

Neglecting rotation, the $f$-mode dispersion relation is ${ }^{6}$

$$
\omega^{2} \approx g \ell / R_{\odot},
$$

where $g$ is the gravitational acceleration at the solar surface. The $m$-degenerate modes are separated by

$$
\Delta v_{\Omega=0}=v_{\ell+1}-v_{\ell} \approx \frac{1}{4 \pi}\left(\frac{g}{\ell R_{\odot}}\right)^{1 / 2} .
$$

Solar rotation lifts the $m$-degeneracy among modes of the

5 Subject to the existence of accessible final states.

6 In the limit $\ell \gg 1$. 


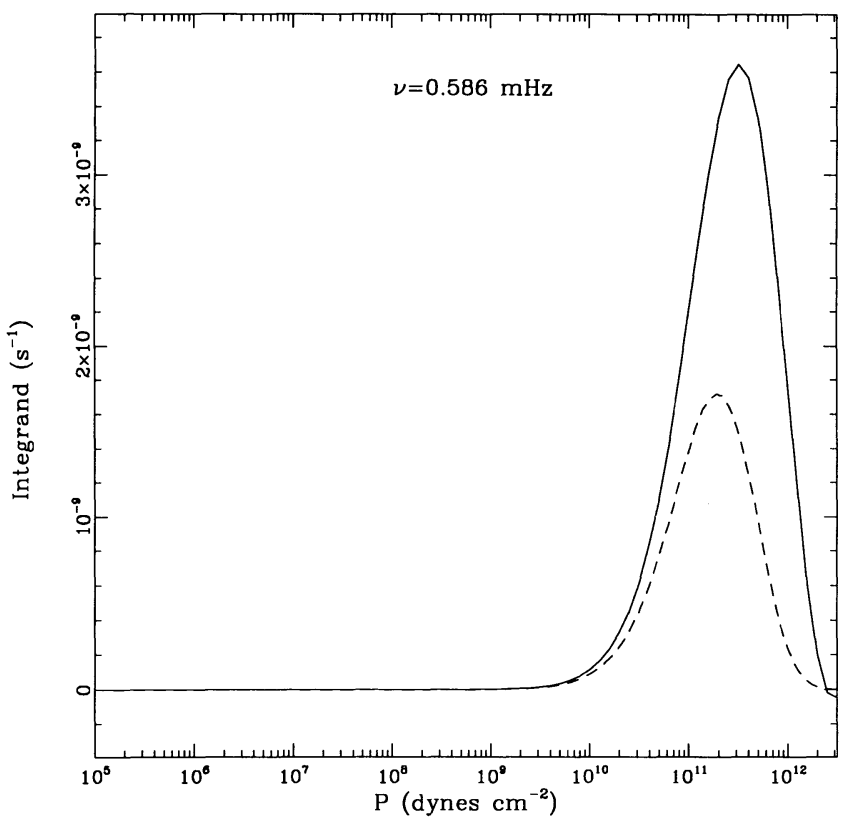

Fig. $3 a$

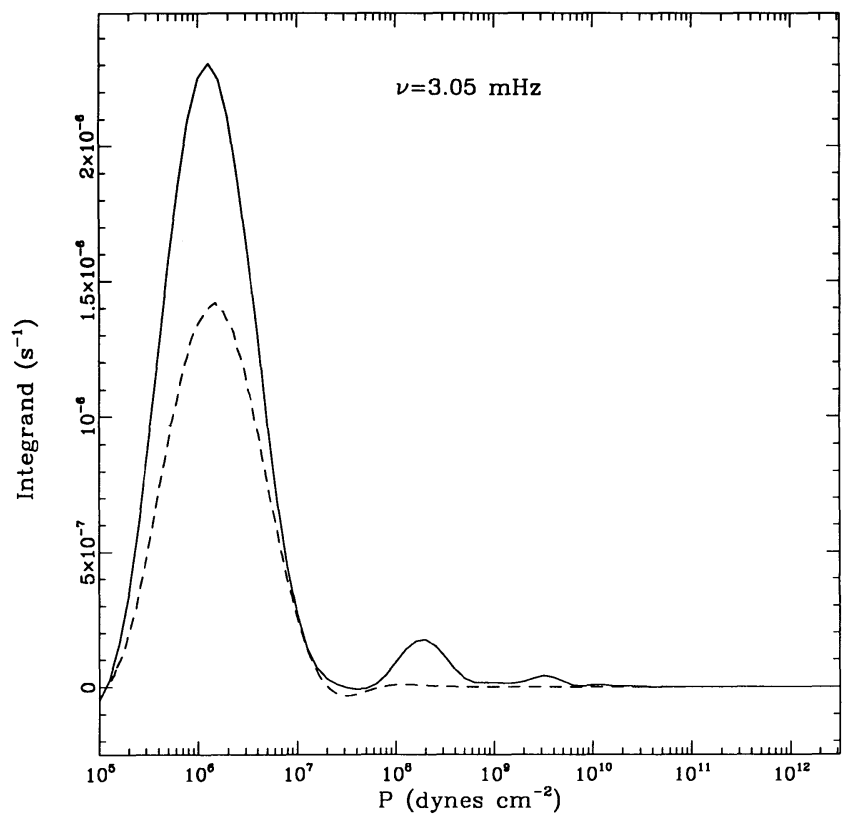

Fig. $3 c$

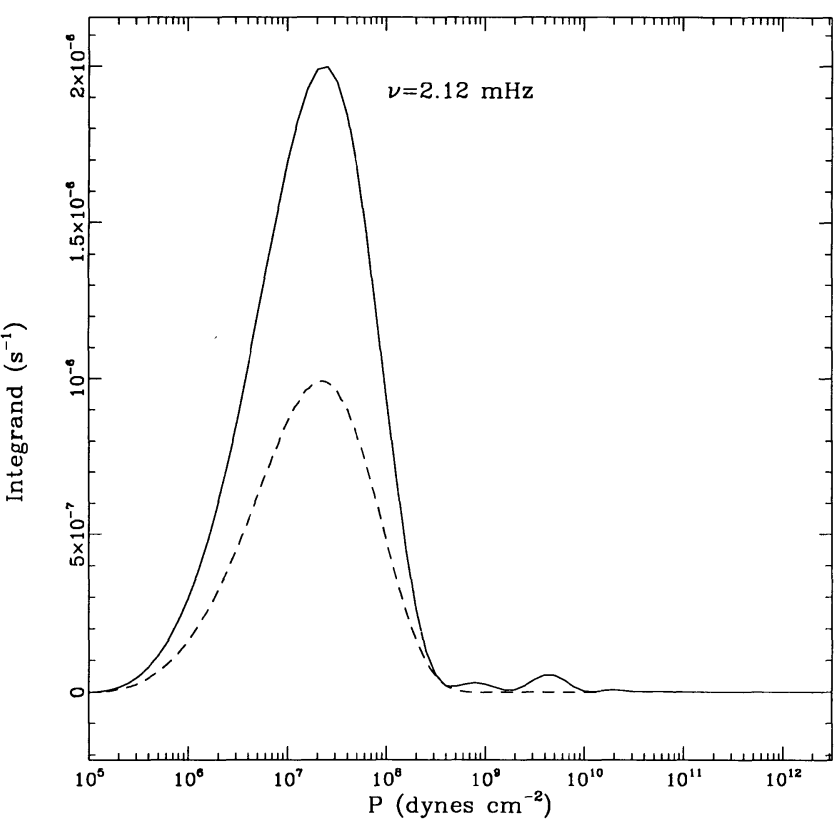

FIG. $3 b$

Fig. 3.-Integrand for $\gamma_{\alpha}^{s}$ obtained by summing over final states $n_{\beta}$, for four different frequencies; (a) $v=0.586 \mathrm{mHz} ;(b) v=2.12 \mathrm{mHz}$; $(c) v=3.05 \mathrm{mHz}$, corresponding to summing the integrands in Fig. 2 , as well as the integrands for $n_{\beta}=4$ to $n_{\beta}=36$; and $(d) v=4.83 \mathrm{mHz}$. For comparison we have also plotted the integrand for scattering into the $f$-mode $\left(n_{\beta}=0\right)$ as the dashed line.

same $\ell$. The rotational splitting can be characterized by

$$
v_{\ell m}-v_{\ell 0}=L \sum_{n} a_{n} P_{n}\left(\frac{m}{\ell}\right),
$$

where $P_{n}$ is the $n$th Legendre polynomial and $L=[\ell(\ell+1)]^{1 / 2}$. The $a_{n}(v, \ell)$ are observationally determined coefficients. For $v \approx 2.5 \mathrm{mHz}$ and $10 \leq \ell \leq 60, a_{1} \approx 440 \mathrm{nHz}$, $a_{2} \approx 5 \mathrm{nHz}$, and $a_{3} \approx 20 \mathrm{nHz}$ (Libbrecht \& Woodard 1991).

Rotational splitting increases the number of different $f$-mode frequencies. However, its effect on the availability of final states is less obvious. Most of the splitting arises from the Sun's mean rotation. It is purely kinematic. Let us consider scattering from a reference frame that revolves with $v=a_{1}$. The principal splitting is this frame is due to $a_{3} \ll a_{1}$. Is $a_{3}$ the relevant splitting affecting the availability of final states? The answer to this question must await a thorough analysis of how the Coriolis acceleration affects scattering in the rotating frame. In particular, it must affect the turbulent velocity field, especially in the lower parts of the convection zone where the eddy lifetimes are comparable to the Sun's rotation period. Unfortunately, such an analysis is outside the scope of the present investigation and 


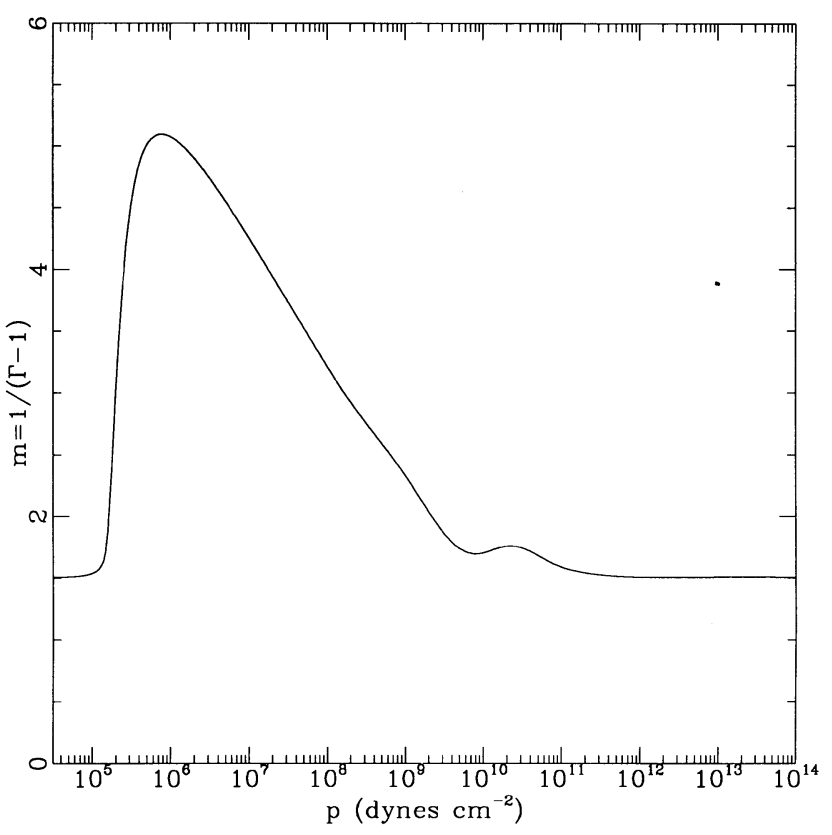

FIG. 4.-Effective polytropic index $m \equiv 1 /\left(\rho c^{2} / p-1\right)$ in the upper layers of the Sun. Note the effect of the hydrogen ionization zone at $p \approx 10^{6}$ dynes $\mathrm{cm}^{-2}$, and the helium ionization zone at $p \approx 3 \times 10^{10}$ dynes $\mathrm{cm}^{-2}$.

must be left for the future. Instead, we examine the consequences of assuming that $a_{3}$ sets the scale of the splitting that is relevant to the availability of final states.

There is a frequency gap between the $(\ell, m=\ell)$ and $(\ell+1$, $m=-\ell-1) f$-modes if

$$
\Delta v_{\Omega \neq 0} \equiv v_{\ell+1,0}-v_{\ell, 0}-(2 \ell+1) a_{3} \geq 0 \text {. }
$$

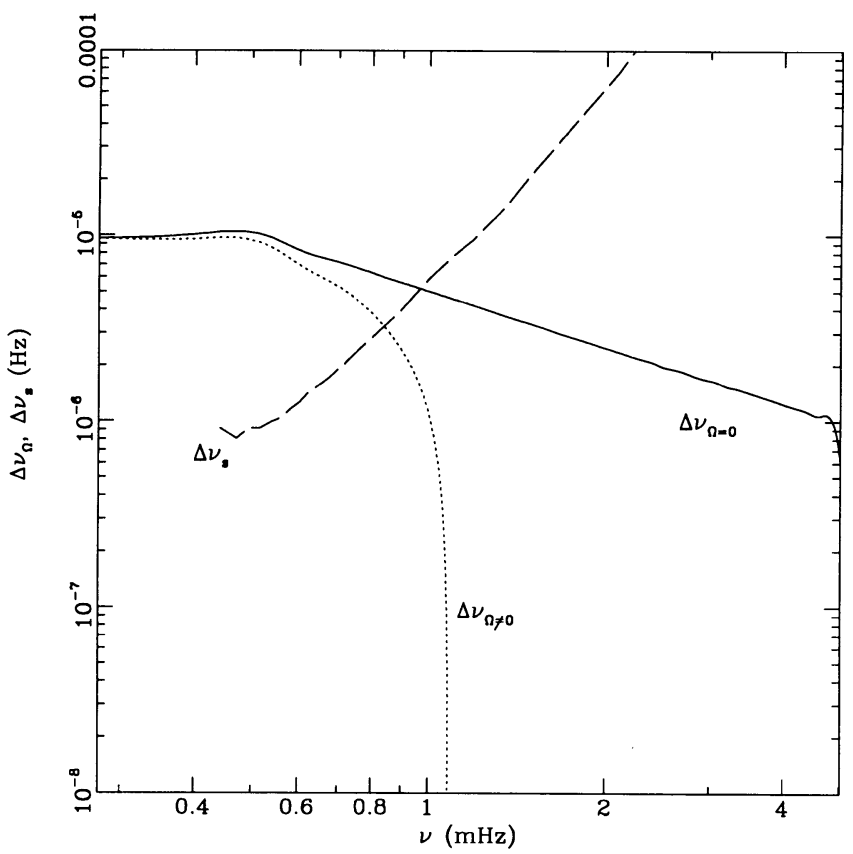

FIG. 5.-Frequency shift $\Delta v_{s}$ associated with scattering (dashed line) together with the spacing in frequency $\Delta v_{\Omega=0}$ between adjacent $f$-modes in a nonrotating solar model (solid line). When the spacing between modes is larger than the frequency shift, here below about $1 \mathrm{mHz}$, there is a dearth of finalstate scattering partners. The dotted line shows $\Delta v_{\Omega \neq 0}$ as estimated by assuming that only $a_{3} \equiv 20 \mathrm{nHz}$ contributes to the splitting between different $m$-modes. See text below eq. (40).
If $\Delta v_{\Omega \neq 0} \leq 0$, the spacing between consecutive $f$-modes is no larger than $a_{3}$. In this case, all $p$-modes have $f$-mode scattering partners, since $a_{3}$ is much smaller than $\Delta v_{s}$ for all $v$.

Figure 5 presents graphs of $\Delta v_{s}, \Delta v_{\Omega=0}$, and $\Delta v_{\Omega \neq 0}$ as functions of $v$. Both $\mathscr{M}_{1}$ and the $f$-mode frequencies are derived from the solar model of Christensen-Dalsgaard (1982). We use $a_{3}=20 \mathrm{nHz}$ in computing $\Delta v_{\Omega \neq 0}$. An accurate calculation would require use of a frequency-dependent $a_{3}$. We observe that final $f$-mode scattering states are available for $p$-modes having $v \gtrsim 1 \mathrm{mHz}$. Rotational splitting, as we have included it, has only a small effect.

As defined above, the absence of final states signals a weakening but not a complete disappearance of scattering. Some scattering always occurs above the top of the acoustic cavity in layers where the correlation time of the turbulent velocity field is sufficient short to ensure the availability of final $f$-mode scattering states.

\section{DISCUSSION}

Scattering couples modes of similar frequency and widely differing angular degree. It drives their energies toward equality. ${ }^{7}$ Under the influence of scattering, absorption, and stochastic excitation, the mode energies evolve according to

$$
\frac{d E_{\alpha}}{d t}=\sum_{n_{\beta}} \gamma_{\alpha \beta}^{s} \Delta N_{\beta}\left(E_{\beta}-E_{\alpha}\right)-\gamma_{\alpha}^{a} E_{\alpha}+\mathscr{P}_{\alpha} .
$$

Here, the sum is over all radial orders $n_{\beta}$, and $\mathscr{P}_{\alpha}$ is the stochastic excitation rate (Goldreich, Murray, \& Kumar 1994, hereafter GMK).

Because mode energies decline with decreasing $n$ at fixed $\omega$ (Kaufman 1990; Rhodes et al. 1990; Fernandes et al. 1992; Willette 1993), ${ }^{8}$ scattering transfers energy from low- $\ell$ to high- $\ell$ modes. Moreover, since $\gamma_{\alpha \beta}^{s} \propto\left(n_{\beta}+1\right)^{-1}$, and $\Delta N_{\beta} \propto$ $\ell_{\beta}^{2}$, scattering couples any given mode $\alpha$ much more strongly to modes of higher rather than lower $\ell$. Thus, scattering acts to damp $p$-modes. The $f$-modes are the ultimate recipients of energy scattered out of the $p$-modes. Hence, scattering acts an excitatory mechanism for $f$-modes. The $p$-modes appear to be stochastically excited, primarily by entropy fluctuations, with fluctuations of the Reynolds stress playing a secondary role (GMK). Because they are nearly imcompressible, $f$-modes are not excited by entropy fluctuations, although they are subject to driving by Reynolds stress fluctuations. It is possible that scattering dominates the damping of $p$-modes and the excitation of $f$-modes.

Unfortunately, the properties of turbulent convection cannot be accurately characterized. The results of the best calculations of which we are capable are still crude, and the relative contributions made by scattering and adsorption to mode line widths remain uncertain.

The authors are indebted to J. Christensen-Dalsgaard for providing them with his solar models, and to P. Kumar and K. Libbrecht for helpful advice. This research was supported by NSF grant AST 89-13664 and NASA grant NAGW 1303.

\footnotetext{
${ }^{7}$ We neglect the fine distinction between action and energy conservation and treat the scattering as elastic.

8 The magnitude of this effect is rather uncertain.
} 


\section{APPENDIX}

Previous calculations of the scattering of sound waves by turbulence (Lighthill 1953; Kraichnan 1953) employ heuristic forms of Eulerian perturbation theory. The results fail to conserve wave action but instead conserve acoustic energy. The conservation of wave action is the classical equivalent of the quantum mechanical conservation of the number of quanta. A mode's action is equal to its energy divided by its frequency. Because the frequency shifts associated with scattering are small, the distinction between the conservation of wave action and the conservation of energy is not a crucial one in our investigation. By working with an action principle, we build the conservation of wave action into our derivation. However, as a consequence of the lack of an adequate theory for turbulence, it still lacks rigor.

We begin by calculating the rate of change of the amplitude of mode $\alpha$ due to its interactions with mode $\beta$ in the presence of the turbulent velocity field. From the amplitude equation we proceed to evaluate the rate at which uncorrelated scatterings cause modes $\alpha$ and $\beta$ to exchange energy. Mode $\alpha$ 's scattering line width is then obtained by summing the exchange rates over all accessible modes $\beta$.

We employ a Lagrangian formulation of fluid dynamics with each fluid element labeled by its background position $\boldsymbol{x}_{\mathbf{0}}$. The Lagrangian displacement, $\boldsymbol{\xi}\left(\boldsymbol{x}_{0}\right)$, associated with waves and turbulence satisfies $\boldsymbol{x}\left(\boldsymbol{x}_{0}\right)=\boldsymbol{x}_{0}+\boldsymbol{\xi}\left(\boldsymbol{x}_{0}, t\right)$. The corresponding Lagrangian and its density are given by

$$
L=\int d^{3} x_{0} \mathscr{L}
$$

and

$$
\mathscr{L}=\frac{1}{2} \rho_{0}\left|\frac{\partial \xi}{\partial t}\right|^{2}-\rho_{0} e(s, \rho)-\rho_{0} \psi
$$

where $e$ is the internal energy density, $s$ is the specific entropy, and $\psi$ is the gravitational potential (Newcomb 1962).

We wish to calculate the scattering to lowest order in the turbulent Mach number $\mathscr{M}$. To this order, the specific entropy is not affected by either the waves or the turbulence; the entropy variations associated with turbulent convection are of order $\mathscr{M}^{2}$, and the waves are adiabatic. Thus, we hold the specific entropy constant when expanding the Lagrangian density in powers of the Lagrangian displacement. With these specifications the second- and third-order Lagrangian densities read

$$
\mathscr{L}_{2}=\frac{\rho}{2}\left|\frac{\partial \xi}{\partial t}\right|^{2}-\frac{\rho c^{2}-p}{2}(\nabla \cdot \xi)^{2}-\frac{p}{2} \xi^{i, j} \xi^{j, i}-\frac{\rho}{2} \psi_{, i j} \xi^{i} \xi^{j},
$$

and

$$
\mathscr{L}_{3}=-\frac{1}{6}\left(\rho c^{2}-p-\left.\rho^{2} \frac{\partial c^{2}}{\partial \rho}\right|_{s}\right)(\nabla \cdot \xi)^{3}+\frac{\rho c^{2}-p}{2}(\nabla \cdot \xi) \xi^{i, j} \xi^{j, i}+\frac{p}{3} \xi^{i, j} \xi^{j, k} \xi^{k, i}-\frac{\rho}{6} \psi_{, i j k} \xi^{i} \xi^{j} \xi^{k} .
$$

All derivatives are taken with respect to $x_{0}$; for example, $\xi^{i, j}=\partial \xi^{i} / \partial x_{0}^{j} .{ }^{9}$ We discard the $\psi$ terms, since the solar gravity is nearly constant throughout the convection zone. Each displacement is the sum of two terms, $\xi=\xi_{w}+\xi_{T}$, respresenting waves and turbulence, respectively.

The Euler-Lagrange equation based on $\mathscr{L}_{2}$ governs the normal modes. It reads

$$
\rho \frac{\partial^{2} \xi}{\partial t^{2}}-\nabla\left[c^{2} \nabla \cdot(\rho \xi)+\rho \xi \cdot g-c^{2} \rho \xi \cdot \nabla \ln \rho\right]+g \nabla \cdot(\rho \xi)=0
$$

The lowest order mode interactions are obtained from $\mathscr{L}_{3}$. We expand $\xi_{w}$ in terms of the normal modes:

$$
\xi_{\alpha}=\frac{1}{\sqrt{2}}\left[A_{\alpha}(t) \xi_{\alpha}(x) e^{-i \omega_{\alpha} t}+A_{\alpha}^{*}(t) \xi_{\alpha}^{*}(x) e^{i \omega_{\alpha} t}\right] .
$$

With the normalization adopted in equation (26), $\left|A_{\alpha}\right|^{2}$ is the energy, $E_{\alpha}$, in mode $\alpha$.

We vary $A_{\alpha}(t)$ in equations (A3) and (A4) to obtain the (complex) amplitude equation

$$
\frac{d A_{\alpha}}{d t}=-M_{\alpha \beta} A_{\beta}
$$

where

$$
\begin{aligned}
M_{\alpha \beta}= & \frac{i \omega_{\alpha}}{\sqrt{2}} e^{-i \Delta \omega t} \int d^{3} x \xi_{T}^{k}\left\{\frac{\partial}{\partial x^{k}}\left[\left(\rho c^{2}-p-\left.\rho^{2} \frac{\partial c^{2}}{\partial \rho}\right|_{s}\right) \xi_{\alpha}^{i, i * \xi_{\beta}^{j, j}}-\left(\rho c^{2}-p\right) \xi_{\alpha}^{i, j * \xi_{\beta}^{j, i}}\right]\right. \\
& \left.-\frac{\partial}{\partial x^{i}}\left[\left(\rho c^{2}-p\right)\left(\xi_{\alpha}^{j, j * \xi_{\beta}^{i, k}}+\xi_{\alpha}^{i, k * \xi_{\beta}^{j, j}}\right)+p\left(\xi_{\alpha}^{j, k * \xi_{\beta}^{i, j}}+\xi_{\alpha}^{i, j * \xi_{\beta}^{j, k}}\right)\right]\right\}
\end{aligned}
$$

\footnotetext{
${ }^{9}$ Henceforth, we omit the subscript zero on $\boldsymbol{x}$.
} 
where $\Delta \omega \equiv \omega_{\beta}-\omega_{\alpha}$. Since

$$
\frac{M_{\alpha \beta}}{\omega_{\alpha}}=-\frac{M_{\beta \alpha}^{*}}{\omega \beta},
$$

scattering conserves wave action.

We do not treat the turbulent dynamics; $\xi_{T}(x, t)$ is taken to be an externally specified, time-variable vector field. Since the turbulent motions are nearly anelastic, $\nabla \cdot\left(\rho \xi_{T}\right) \approx 0$, we express $\rho \xi_{T}$ in terms of a vector potential $\mathscr{A}$, as

$$
\rho \xi_{T}=\nabla \times \mathscr{A} \text {. }
$$

In addition to explicitly accounting for the vanishing divergence of $\rho \xi_{T}$, this step enables us to integrate by parts and then apply the equation of motion (A5) to transform $M_{\alpha \beta}$ into

$$
\begin{aligned}
M_{\alpha \beta}= & \frac{i \omega_{\alpha}}{\sqrt{2}} e^{-i \Delta \omega t} \int d^{3} x \epsilon_{k \ell m} \mathscr{A}_{m}\left\{\left(\omega_{\beta}^{2}-\omega_{\alpha}^{2}\right) \xi_{\alpha}^{i, \ell * \xi_{\beta}^{i, k}}+\left[\frac{1}{\rho} \frac{\partial}{\partial x^{\ell}}\left(\rho c^{2}-p\right)-\frac{\partial c^{2}}{\partial x^{\ell}}\right] \frac{\partial}{\partial x^{k}}\left(\xi_{\alpha}^{i, i * \xi_{\beta}^{j, j}}-\xi_{\alpha}^{i, j * \xi_{\beta}^{j, i}}\right)\right. \\
& \left.+\left[\frac{\partial c^{2}}{\partial x^{\ell}}-\left.\frac{\partial c^{2}}{\partial \rho}\right|_{s} \frac{\partial \rho}{\partial x^{\ell}}\right] \frac{\partial}{\partial x^{k}}\left(\xi_{\alpha}^{i, i * \xi_{\beta}^{j, j}}\right)\right\},
\end{aligned}
$$

where $\epsilon_{k \ell m}$ is the completely antisymmetric tensor. The frequency difference in the first term is a consequence of the antisymmetry combined with the action of the two derivatives $\partial_{k}$ and $\partial_{\ell}$ on the wave displacements. The remaining terms arise from the action of $\partial_{\ell}$ on the thermodynamic variables. They are proportional to $\partial s / \partial r$, and thus vanish for an isentrope.

Now we assess the relative magnitudes of the three terms in equation (A11). The coefficient of the first term is

$$
\left|\omega_{\beta}^{2}-\omega_{\alpha}^{2}\right| \approx 2 \omega|\Delta \omega| \lesssim \frac{\omega}{\tau} \sim \frac{\omega v}{H}
$$

The coefficients of the second and third terms can be related to the entropy gradient by

$$
\left[\frac{1}{\rho} \frac{\partial}{\partial r}\left(\rho c^{2}-p\right)-\frac{\partial c^{2}}{\partial r}\right]=-\frac{c^{2}}{c_{p}} \frac{d s}{d r} \sim \frac{v^{2}}{H}
$$

and

$$
\left[\frac{\partial c^{2}}{\partial r}-\left.\frac{\partial c^{2}}{\partial \rho}\right|_{s} \frac{\partial \rho}{\partial r}\right]=\left.\frac{\partial c^{2}}{\partial s}\right|_{p} \frac{d s}{d r} \sim-\frac{v^{2}}{H}
$$

The final forms of equations (A13) and (A14) follow from the mixing-length relation

$$
\frac{1}{c_{p}} \frac{d s}{d r} \sim \frac{\mathscr{M}^{2}}{H},
$$

where $c_{p}$ is the specific heat at constant pressure. Since the gradient operator acting on $\xi$ has magnitude $\lesssim \omega / c$, the second and third terms are smaller than the first by a factor of order $\mathscr{M}$. In what follows, we retain only the first term.

The expectation value of the rate of change of $E_{\alpha}$ due to energy scattered from and into mode $\beta$ involves an ensemble average over a large number of uncorrelated scatterings off many different turbulent eddies. Thus we integrate equation (A7) over a time $\delta t$ such that $\tau_{\boldsymbol{H}} \ll \delta t \ll 1 / \gamma_{\alpha}$. To second order in $\delta t$, we find

$$
A_{\alpha}(t+\delta t)=A_{\alpha}-\int_{t}^{t+\delta t} d t^{\prime} M_{\alpha \beta}\left(t^{\prime}\right) A_{\beta}(t)+\int_{t}^{t+\delta t} d t^{\prime} \int_{t}^{t^{\prime}} d t^{\prime \prime} M_{\alpha \beta}\left(t^{\prime}\right) M_{\beta \alpha}\left(t^{\prime \prime}\right) A_{\alpha}(t) .
$$

Subtracting $\left|A_{\alpha}(t)\right|^{2}$ from $\left|A_{\alpha}(t+\delta t)\right|^{2}$ yields

$$
\Delta E_{\alpha}=\int_{t}^{t+\delta t} d t^{\prime} M_{\alpha \beta}^{*}\left(t^{\prime}\right) \int_{t}^{t+\delta t} d t^{\prime \prime} M_{\alpha \beta}\left(t^{\prime \prime}\right)\left[E_{\beta}(t)-E_{\alpha}(t)\right]-\int_{t}^{t+\delta t} d t^{\prime} M_{\alpha \beta}\left(t^{\prime}\right) A_{\alpha}^{*}(t) A_{\beta}(t)-\int_{t}^{t+\delta t} d t^{\prime} M_{\alpha \beta}^{*}\left(t^{\prime}\right) A_{\alpha}(t) A_{\beta}^{*}(t) .
$$

Taking the ensemble average and making the random phase approximation, we arrive at

$$
\gamma_{\alpha \beta} \equiv \frac{1}{E_{\beta}-E_{\alpha}}\left\langle\frac{d E_{\alpha}}{d t}\right\rangle=\frac{1}{\delta t} \int_{t}^{t+\delta t} d t^{\prime} \int_{t}^{t+\delta t} d t^{\prime \prime}\left\langle M_{\alpha \beta}^{*}\left(t^{\prime}\right) M_{\alpha \beta}\left(t^{\prime \prime}\right)\right\rangle
$$

The next step is to substitute the expression given by equation (A11) for $M_{\alpha \beta}$ in equation (A18). Before doing so, we introduce a number of simplifying approximations. We take mode $\alpha$ to be of low degree and mode $\beta$ to be of high degree, and treat both in a quasi-plane-parallel manner. That is, we erect a local Cartesian coordinate system with the $z$-axis aligned in the radial direction. 
Then we factor out the angular dependence of the eigenfunctions by writing

$$
\xi_{\alpha}(x)=\exp \left(i k_{\alpha} \cdot x\right) \tilde{\xi}_{\alpha}(r)
$$

Here $\boldsymbol{k}_{\alpha}$ is a horizontal wavevector; $k_{\alpha} \equiv \ell_{\alpha} / \boldsymbol{R}_{\odot}$.

It is felicitous to employ the physical variable $\rho v$ rather than $\mathscr{A}$. To do so, we integrate by parts with respect to $t^{\prime}, t^{\prime \prime}, \boldsymbol{x}^{\prime}$, and $\boldsymbol{x}^{\prime \prime}$. The spatial integrations require some care. We have assumed that $\boldsymbol{k}_{\alpha} \ll \boldsymbol{k}_{\beta}$, so we remove the spatial derivatives from $\xi_{\beta}$ and apply them to $\mathscr{A} .^{10}$ The result reads

$$
\gamma_{\alpha \beta}=\frac{1}{\delta t} \int_{t}^{t+\delta t} d t^{\prime} \int_{t}^{t+\delta t} d t^{\prime \prime} \int d^{3} x^{\prime} \int d^{3} x^{\prime \prime} e^{-i \Delta \omega\left(t^{\prime \prime}-t^{\prime}\right)} \times\left\langle\rho v_{m}\left(x^{\prime}, t^{\prime}\right) \rho v_{n}\left(x^{\prime \prime}, t^{\prime \prime}\right)\right\rangle G_{m n}\left(x^{\prime}, x^{\prime \prime}\right),
$$

where the dominant term in $G_{m n}$, expressed in dyadic notation, appears as

$$
\boldsymbol{G}\left(\boldsymbol{x}^{\prime}, \boldsymbol{x}^{\prime \prime}\right) \approx 2 \omega^{4} \exp \left[i \boldsymbol{k}_{\beta} \cdot\left(\boldsymbol{x}^{\prime \prime}-\boldsymbol{x}^{\prime}\right)\right] \frac{\partial \tilde{\xi}_{\alpha}^{r}}{\partial r^{\prime}}\left(r^{\prime}\right) \frac{\partial \tilde{\xi}_{\alpha}^{r}}{\partial r^{\prime \prime}}\left(r^{\prime \prime}\right) \tilde{\xi}_{\beta}^{r}\left(r^{\prime}\right) \tilde{\xi}_{\beta}^{r}\left(r^{\prime \prime}\right) \hat{\boldsymbol{e}}_{r} \hat{\boldsymbol{e}}_{r}
$$

Here $\hat{\boldsymbol{e}}_{\boldsymbol{r}}$ is the radial unit vector. Since the dominant components of $\boldsymbol{G}$ are vertical, only the vertical components of $\left\langle\rho v_{m} \rho v_{n}\right\rangle$ are relevant to the computation of $\gamma_{\alpha \beta}$.

We choose

$$
\left\langle\rho v_{r}\left(x^{\prime}, t^{\prime}\right) \rho v_{r}\left(x^{\prime \prime}, t^{\prime \prime}\right)\right\rangle=\rho^{2} v^{2}(\bar{r}) \exp \left\{-\pi\left[\left(\frac{\Delta t}{\tau}\right)^{2}+\left(\frac{\Delta r}{\Lambda}\right)^{2}+\left(\frac{\left|\Delta x_{h}\right|}{\mathscr{S} \Lambda}\right)^{2}\right]\right\}
$$

as the correlation function for the product of the density and the turbulent velocity field. This form is adopted for its simplicity, and because $\exp \left(-\pi x^{2}\right)$ integrates to unity. In the above and in what follows, we split the spacetime variables into average values and differences according to $\bar{t} \equiv\left(t^{\prime}+t^{\prime \prime}\right) / 2, \Delta t \equiv t^{\prime \prime}-t^{\prime}, \bar{r} \equiv\left(r^{\prime}+r^{\prime \prime}\right) / 2, \Delta r \equiv r^{\prime \prime}-r^{\prime}, \bar{x}_{h} \equiv\left(x_{h}^{\prime}+x_{h}^{\prime \prime}\right) / 2$, and $\Delta x_{h} \equiv x_{h}^{\prime \prime}-x_{h}^{\prime}$, where $x_{h}$ denotes the horizontal part of $x$. Note that $\tau, \Lambda, \mathscr{S}, v$ and $\rho$ are functions of $\bar{r}$.

Equation (A20) for $\gamma_{\alpha \beta}$ involves an eight-dimensional integral. Six of these integrations can be carried out analytically. Those over $\Delta t$ and $\Delta x_{h}$ are just Fourier transforms. When they are completed, $\bar{t}$ and $\bar{x}_{h}$ no longer appear in the integrand, so the integrations over these variables are trivial. To sum the scattering rate over all modes with radial order $n_{\beta}$, we first average the direction of $v$ with respect to the vertical, which yields a factor $\frac{1}{2}$. Then we multiply the expression for $\gamma_{\alpha \beta}$ by the density of modes on ridge $\beta$,

$$
\frac{d N_{\beta}}{d \omega}=2 R_{\odot}^{2} k_{\beta} \frac{d k_{\beta}}{d \omega},
$$

and integrate over $\Delta \omega$ to find

$$
\gamma_{\alpha \beta} \Delta N_{\beta} \approx 16 \pi^{2} R_{\odot}^{4} \omega^{4} k_{\beta} \frac{d k_{\beta}}{d \omega} \int_{r_{b}}^{R_{\odot}} d \bar{r} \rho^{2} v_{\Lambda}^{2} \mathscr{S}^{2} \Lambda^{2} \exp \left[-\frac{\left(k_{\beta} \mathscr{S} \Lambda\right)^{2}}{4 \pi}\right] \int_{\Delta r_{\ell}}^{\Delta r_{u}} d \Delta r \frac{\partial \tilde{\xi}_{\alpha}^{r^{\prime}}}{\partial r^{\prime}} \frac{\partial \tilde{\xi}_{\alpha}^{r^{\prime \prime}}}{\partial r^{\prime \prime}} \tilde{\xi}_{\beta}^{r^{\prime}} \tilde{\xi}_{\beta}^{r^{\prime \prime}} \exp \left[-\pi\left(\frac{\Delta r}{\Lambda}\right)^{2}\right],
$$

where the upper and lower limits on the second integral are $\pm 2\left(\bar{r}-r_{b}\right)$ if $r_{b} \leq \bar{r} \leq\left(r_{b}+R_{\odot}\right) / 2$, and $\pm 2\left(\mathscr{R}_{\odot}-\bar{r}\right)$ if $\left(r_{b}+R_{\odot}\right) / 2 \leq$ $\bar{r} \leq R_{\odot}$, and $r_{b}$ denotes the base of the convection zone. The upper sign refers to $\Delta r_{u}$, the lower sign to $\Delta r_{\ell}$. Equation (A24) is used in numerical evaluations of the scattering line width.

We gain insight into the scattering process by evaluating equation (A24) analytically. An analysis of the equation of motion (eq. [A5]) shows that the modes propagate between an upper turning point at $r_{1}$, defined by $\omega / c \approx \frac{1}{2} \partial \ln \left(\rho c^{2} / r^{2}\right) / \partial r \equiv 1 /(2 \hat{H})$, and a lower turning point at $r_{2}$, defined by $\omega^{2} / c^{2} \approx \ell(\ell+1) / r^{2}$. Figure 6 displays the ratio $H / \hat{H}$ as a function of $\rho$. Note the effect of the ionization zone. A WKB analysis yields

$$
\tilde{\xi}^{r} \approx B \frac{r_{1}}{r}\left(\frac{\rho_{1} c_{1}}{\rho c}\right)^{1 / 2} \cos \left(\int_{r}^{r_{1}} \frac{\omega}{c} d r+\phi\right)
$$

between $r_{1}$ and $r_{2}$, where $\phi$ is a constant. Below $r_{2}, \tilde{\xi}^{r} \propto\left(r / r_{2}\right)^{\ell}$, whereas above $r_{1}$ the regular solution is

$$
\tilde{\xi} \approx B \exp \left(\int_{r_{1}}^{r} \frac{\omega^{2} \hat{H}}{c^{2}} d r\right) .
$$

We note for later use that this implies $\partial \tilde{\xi} / \partial r \approx\left(\omega^{2} \hat{H} / c^{2}\right) \tilde{\xi}$ above $r_{1}$. We find $B$ from the normalization condition (eq. [26]):

$$
B^{2} \approx \frac{1}{2 \pi^{2} R_{\odot}^{2}(n+1) \omega \rho_{1} c_{1}} .
$$

${ }^{10}$ We could also remove the derivatives from $\xi_{\alpha}$, but that would result in an integral having substantial cancellation the value of which could not be estimated from the absolute value of its integrand. 


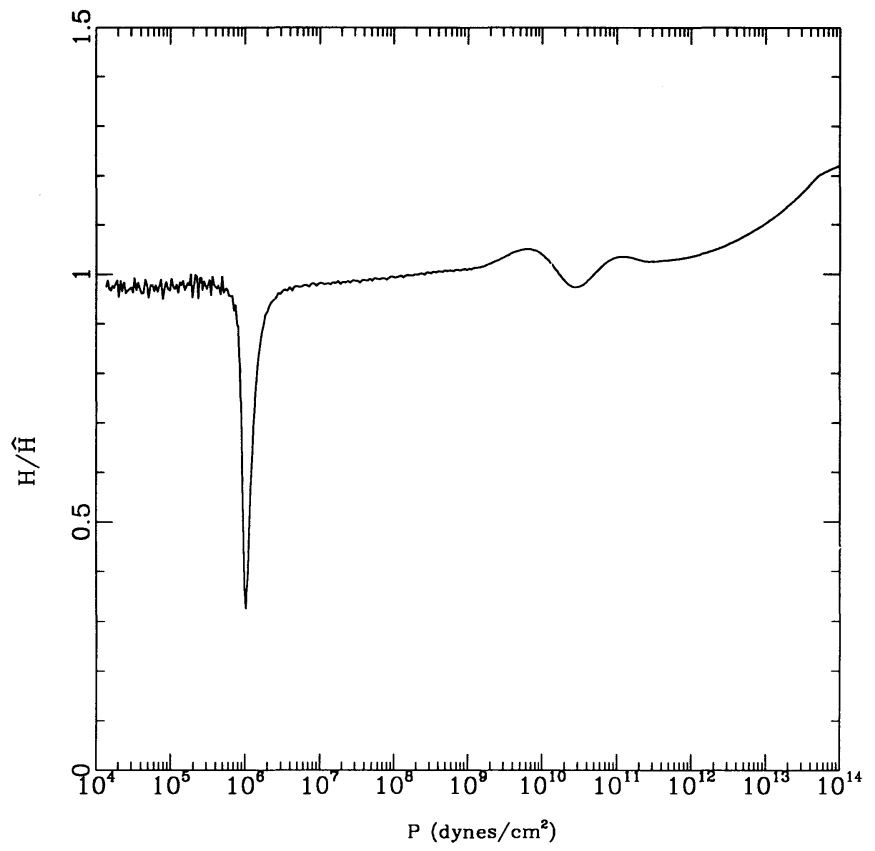

Fig. 6.-Ratio of the pressure scale height $H$ to $\hat{H} \equiv \partial \ln \left(\rho c^{2} / r^{2}\right) / \partial r$, demonstrating that $H \approx \hat{H}$ over most of the convection zone

Most of the normalization integral comes from the acoustic cavity between $r_{1}$ and $r_{2}$. The 1 in the factor $n+1$ accounts in rough fashion for the contribution from the evanescent region above $r_{1}$.

The evaluation of the integral over $\Delta r$ in equation (A24) is straightforward; we average over the oscillations in the acoustic cavity and treat the exponential factor as a delta function. With a little manipulation, equations (A24), (A25), and (A26) yield

$$
\begin{aligned}
\gamma_{\alpha \beta} \Delta N_{\beta} \approx & \frac{\omega^{4} k_{\beta}}{2 \pi^{2}\left(n_{\alpha}+1\right)\left(n_{\beta}+1\right)} \frac{d k_{\beta}}{d \omega} \int_{r_{b}}^{R_{\odot}} \frac{d \bar{r}}{\Lambda}\left(\frac{r_{1}}{r}\right)^{4} \frac{v_{\Lambda}^{2} \mathscr{S}^{2} \Lambda^{4}}{c^{4}} \exp \left[-\frac{\left(k_{\beta} \mathscr{S} \Lambda\right)^{2}}{4 \pi}\right] \\
& \times \begin{cases}2\left(\frac{\rho c}{\rho_{1} c_{1}}\right)^{2}\left(\frac{2 \omega \hat{H}}{c}\right)^{2}\left(\frac{\bar{r}}{r_{1}}\right)^{4} \exp \left(2 \int_{r_{1}}^{\bar{r}} \frac{\omega^{2} \hat{H}}{c^{2}} d r\right), & r_{1}<\bar{r}<R_{\odot}, \\
1, & r_{2}<\bar{r}<r_{1}, \\
0, & r_{b}<\bar{r}<r_{2} .\end{cases}
\end{aligned}
$$

The integrand increases monotonically with depth until cutoff either by the exponential factor or by $r_{2}$ of mode $\beta$.

To assess the contribution of a single layer of eddies to $\gamma_{\alpha}^{s}$, we sum the integrand in equation (A24) over $n_{\beta}$. We make use of the approximate dispersion relation $\omega^{2} \approx g k(n+1)$, set $r / r_{1}=1, c^{2} / \Lambda=g$, and ignore the cutoff associated with $r_{2} .{ }^{11}$ Together, these approximations lead to

$$
\gamma_{\alpha}^{s} \approx \frac{2 \omega^{3}}{\pi\left(n_{\alpha}+1\right) g^{2}} \int_{r_{b}}^{R_{\odot}} \frac{d \bar{r}}{\Lambda} v_{\Lambda}^{2} \times \begin{cases}2\left(\frac{\rho c}{\rho_{1} c_{1}}\right)^{2}\left(\frac{2 \omega \hat{H}}{c}\right)^{2} \exp \left(2 \int_{r_{1}}^{\bar{r}} \frac{\omega^{2} \hat{H}}{c^{2}} d r\right), & r_{1}<\bar{r}<R_{\odot}, \\ 1, & r_{b}<\bar{r}<r_{1} .\end{cases}
$$

The above integrand peaks near $r=r_{1}$. An approximate evaluation yields

$$
\gamma_{\alpha}^{s} \approx \frac{2 \omega}{\pi} \frac{\mathscr{M}_{1}^{2}}{\left(n_{\alpha}+1\right)},
$$

in agreement with the heuristic result derived in $\S 2$.

${ }^{11}$ This final approximation results in an overestimate of the scattering to the $f$-mode. 
Ando, H., \& Osaki, Y. 1975, PASJ, 27, 581

Antia, H. M. Chitre, S. M. \& Narasimha, K. 1986, Ap\&SS, 118, 169 Balmforth, N. J. 1992a, MNRAS, 255, 603

. N. J. 1992b, MNRAS, 255, 639

' 2 ' Cattaneo, F., Brummell, N. H., Toomre, J., Malagoli, A., \& Hurlburt, N. E. 1991, ApJ, 370, 282

- Christensen-Dalsgaard, J. 1982, MNRAS, 199, 735

Christensen-Dalsgaard, J., Gough, D. O., \& Libbrecht, K. G. 1989, ApJ, 341, L103

Fernandes, D. N., Scherrer, P. H., Tarbell, R. D., \& Title, A. M. 1992, ApJ, 392, 736

Goldreich, P., \& Keeley, D. A. 1977, ApJ, 211, 934

Goldreich, P., \& Kumar, P. 1988, ApJ, 326, 462

. 1991, ApJ, 374, 366

\section{REFERENCES}

Goldreich, P., Murray, N., \& Kumar, P. 1994, ApJ, 423, 466 (GMK)

Kaufman, J. M. 1990, Ph.D. thesis, California Inst. Technology

Kraichnan, R. H. 1953, J. Acoust. Soc. Am., 25, 1096

Libbrecht, K. G. 1988, ApJ, 334, 510

Libbrecht, K. G., \& Woodard, M. F. 1991, Science, 253, 152

Lighthill, M. J. 1953, Proc. Cambridge Phil. Soc., 49, 531

Newcomb, W. A. 1962, Nucl. Fusion Suppl., 2, 451

Rast, M. P., Nordlund, A., Stein, R. F. \& Toomre, J. 1993, ApJ, 408, 53

Rhodes, E. J., Brown, T. M., Cacciani, A., Korzennik, S. G., \& Ulrich, R. K. 1990, in Lecture Notes in Physics 388, Challenges to Theories of the Structure of Moderate-Mass Stars, ed. D. Gough \& J. Toomre (Berlin: SpringerVerlag), 277

Stein, R. F., \& Nordlund, A. 1989, ApJ, 342, 95

Willette, G. 1993, in preparation 